\title{
Uma Proposta de Metodologia para Avaliação de Mapas Conceituais
}

\author{
Raimundo Nonato Bezerra Neto ${ }^{1}$, Rommel Wladimir de Lima ${ }^{1}$ \\ ${ }^{1}$ Programa de Pós Graduação em Ciência da Computação (PPgCC) \\ Universidade do Estado do Rio grande do Norte (UERN) - Mossoró, RN - Brasil \\ bezerraneto, rommelwladimirauern.br
}

\begin{abstract}
Conceptual Maps are gaining space in the educational environment, this tool allows the student to structure their understanding through diagrams where knowledge is interrelated forming logical propositions, knowledge that will serve as a subsidy for the teacher in the moment of identifying the level of assimilation of the student in a specific content. The objective of this work is to structure a methodology of evaluation of conceptual maps that allows the analysis of its taxonomy, performing the classification of its logical structure of knowledge, resulting in a return with enough information to measure the degree of knowledge of the student in a certain subject, according to this analysis.
\end{abstract}

Resumo. Os Mapas Conceituais vem ganhando espaço no ambiente educacional, essa ferramenta permite que o aluno estruture seu entendimento através de diagramas onde o conhecimento é inter-relacionado formando proposições lógicas, conhecimento esse que servirá de subsídio para o professor no momento de identificar o nível de assimilação do discente em um conteúdo específico. O objetivo deste trabalho é estruturar uma metodologia de avaliação de mapas conceituais que possibilite a análise de sua taxonomia, realizando o ranqueamento da sua estrutura lógica de conhecimento, resultando em um retorno com informações suficientes para mensurar o grau de conhecimento do aluno em um determinado assunto, de acordo com essa análise.

\section{Introdução}

O ensino superior tradicional vem passando por transformações estruturais e metodológicas, mudanças essas que desafiam a forma de ensinar, de aprender e atinge diretamente a sua sustentabilidade. Segundo [Valente 2014] o modelo de universidade que faz pesquisa, gera conhecimento e distribui este conhecimento para poucos, já não se sustenta mais.

Seguindo a concepção de [Trevelin et al. 2013], a inserção de tecnologia no ambiente de ensino aumenta substancialmente a possibilidade de aprendizagem dos alunos, interação com os professores, pessoas, objetos e informações que estejam no mesmo ambiente de ensino, modificando toda a dinâmica de aula.

Apoiado nessa ideia os mapas conceituais vêm tornando-se uma boa opção para representar os modelos mentais idiossincráticos dos indivíduos. Com isso, em meados da década de setenta, Joseph Novak introduziu essa representação gráfica que continha proposições claras e termos de ligação que expressavam as relações conceituais entre essas proposições, tornando um método eficiente de organização do conhecimento. 
VII Congresso Brasileiro de Informática na Educação (CBIE 2018)

Anais do XXIX Simpósio Brasileiro de Informática na Educação (SBIE 2018)

Segundo [Cicuto et al. 2012], a avaliação do conhecimento permite medir os resultados da aprendizagem e determina se o nível do processo educacional foi bemsucedido. Com isso, o objetivo desse trabalho é propor metodologia de avaliação que possa realizar o ranqueamento automático do conhecimento do aluno manifestado na construção de um mapa conceitual, podendo assim facilitar a análise do professor sobre esse conhecimento em determinado conteúdo. Para a realização desse ranqueamento utilizaremos as ideias de Conceitos Obrigatórios e Análise de Vizinhança (CO, AV) de [Cañas et al. 2012] e os critérios de avaliação de mapas conceituais de [Cicuto et al. 2012].

Este resumo está organizado da seguinte forma: A seção 2 expõe a teoria da aprendizagem significativa de David Ausubel, a Seção 3 os critérios de avaliação de mapas conceituais, na Seção 4 é descrito os termos obrigatórios e a análise de vizinhança, na Seção 5 indicaremos a proposta da ferramenta computacional, e por fim, na Seção 6 encerraremos com as conclusões.

\section{Aprendizagem Significativa}

A Teoria da Aprendizagem Significativa proposta por David Ausubel, explica que "O fator isolado mais importante que influencia a aprendizagem é aquilo que o aprendiz já conhece. Descubra o que o aluno sabe e baseie nisso seus ensinamento" [Ausubel et al. 1980].

São três as condições elementares para se aprender de forma significativa: a apresentação de um novo conhecimento formulado de maneira lógica; a presença de conceitos prévios na estrutura cognitiva que possibilite a sua relação com o novo conhecimento, e a ação clara de apreender e unir o seu conhecimento com o que pretende absorver. Esses conhecimentos antecipados são também chamados de conceitos subsunçores ou âncora.

$\mathrm{Na}$ trajetória da aprendizagem significativa os conceitos, que se relacionam com o novo conhecimento adquirido e auxiliam na atribuição de novos significados, vão se modificando em detrimento dessa interação, surgindo novas interpretações e se diferenciando progressivamente. Essa etapa proativa pertencente a estrutura cognitiva dar-se-á o nome de diferenciação progressiva.

Seguindo a mesma trajetória, temos a reconciliação integrativa, procedimento que estabelece relações entre ideias, conceitos, proposições já pactuadas na estrutura cognitiva e relações entre subsunçores. Segundo [Moreira 2012] as reconciliações integrativas são elementos existentes na estrutura cognitiva com determinado grau de clareza, estabilidade, diferenciação e são percebidos como relacionados, adquirem novos significados e levam a uma reorganização da estrutura cognitiva.

\section{Critérios de Avaliação de Mapas Conceituais}

Quando se fala em avaliação de mapas conceituais, pensa-se em uma junção de técnicas que possibilite auxiliar o docente nessa tarefa, algum mecanismo que facilite a pontuação e resulte em um valor a ser justificado pelo docente. Na literatura existem várias rubricas que foram desenvolvidas para classificar, pontuar e compreender mapas conceituais, em sua maioria derivadas de projetos maiores. 
VII Congresso Brasileiro de Informática na Educação (CBIE 2018)

Anais do XXIX Simpósio Brasileiro de Informática na Educação (SBIE 2018)

Dentre os estudos pesquisados podemos citar como relevantes [Cañas et al. 2012] onde traz uma pesquisa bibliográfica sobre o uso de tarefas baseadas em mapas conceituais para fins de avaliação do conhecimento e critérios de pontuação. Analisamos também [Wang et al. 2011] que faz uma classificação entre mapas conceituais, gerando critérios que avaliam esses mapas como "pobre", "bom" e "excelente", além do trabalho já citado de [Cicuto et al. 2012].

Baseando-se nesses estudos, selecionamos as rubricas que mais se aproximaram da nossa pesquisa: Quantidade de Proposições, Qualidade das Proposições e Profundidade da Explicação das Proposições, juntando essas três rubricas com a ideia de conceitos obrigatórios e análise de vizinhança.

\section{Conceitos Obrigatórios e Análise de Vizinhança}

A utilização do conceito de Análise de Vizinhança (AV) é uma metodologia utilizada para aferir a compreensão dos alunos em um determinado conteúdo, afirma [Cicuto et al. 2012]. Essa ideia baseia-se na construção de um mapa conceitual levando em consideração um objetivo específico, aqui chamado de "pergunta focal" e da escolha de termos diretamente ligados a esse objetivo, os "conceitos obrigatórios" que são escolhidos de forma criteriosa pelo professor para criar uma carga cognitiva que auxilie a memorização dos fatos e dados.

De acordo com a versão revisada da taxonomia de Bloom, é possível prever que a elaboração de Mapas Conceituais associado a um Conceito Obrigatório envolve, além de conhecimentos conceituais e procedimentais, processos cognitivos relacionados com análise, avaliação e criação [Krathwohl 2002]. Quando o Conceito Obrigatório (CO) é selecionado de forma cautelosa pelo professor, o esforço cognitivo imposto aos alunos é mais sofisticado do que aquele que é usualmente necessário para obter bom desempenho em provas tradicionais [Cicuto et al. 2012].

Seguindo esse raciocínio, quando o professor introduz a ideia de Conceito Obrigatório $(\mathrm{CO})$ na criação dos mapas conceituais, reforça, no aluno, a possibilidade deste aprender de forma significativa, retomando a ideia de David Ausubel.

Com a inserção do $\mathrm{CO}$ pelo professor, podemos classificar os demais termos adicionados inseridos pelo aluno como Conceitos Vizinhos (CV), que estão diretamente ligados com os CO's e Conceitos Complementares (CC), esses não estão intimamente relacionados com os CO's. Sendo assim, podemos inferir que quanto mais (CV) o mapeador utilizar, mais ligado aos (CO's) e, por conseguinte, mais próximo da pergunta focal, sendo um dos pontos de análise para classificar o mapa conceitual. Essa trajetória de conceitos obrigatórios com conceitos vizinhos retoma a proposta de David Ausubel de diferenciação progressiva, gerando o relacionamento dos conceitos existentes as novas concepções.

\section{Proposta}

Esse artigo é parte introdutória do desenvolvimento de uma metodologia que visa ranquear mapas conceituais levando em consideração rubricas específicas. Dividimos a proposta em três partes: Criação do Mapa Conceitual, Análise dos Dados e Ranqueamento. A observação da Figura 1 remonta essa fase de construção da ferramenta. 


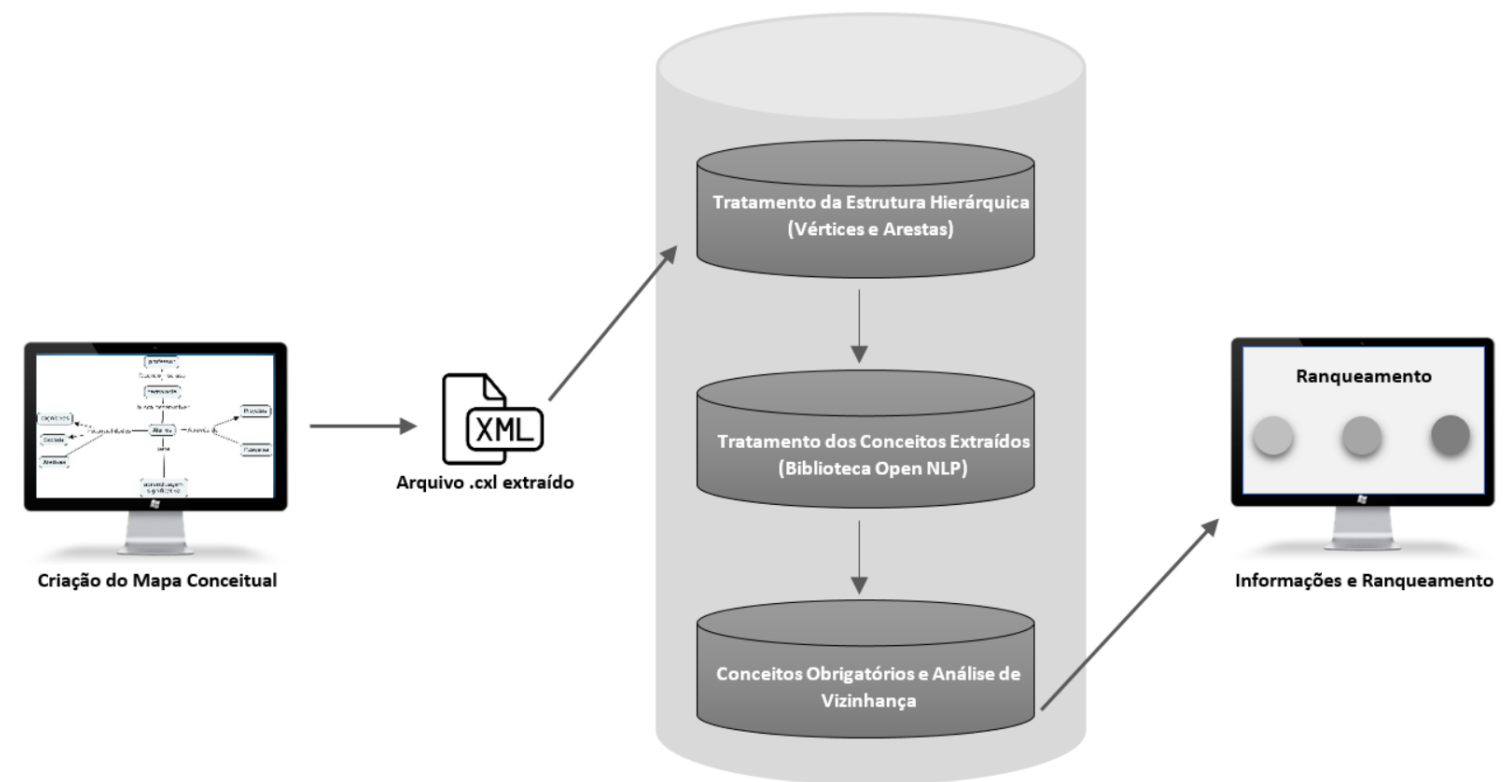

Figura 1. Fase de Criação do Mapa Conceitual, Exportação, Análise dos Dados e Ranqueamento

- Criação do Mapa Conceitual: A primeira etapa consistirá da criação da atividade pelo docente. O professor irá compartilhar com os alunos o tema em questão, elaborado a pergunta focal e definindo seu domínio. É nessa fase que os Conceitos Obrigatórios são elencados, o professor irá expor esses conceitos que foram criteriosamente escolhidos com base no conteúdo abordado. Os alunos irão desenvolver o mapa conceitual na ferramenta CMapTools, no qual o resultado desse trabalho deve ser exportado para a nossa ferramenta em formato cxl, um schema $x m l$ utilizado pelo CMapTools. Por se tratar de uma ferramenta Web, seu desenvolvimento será em linguagem de programação PHP.

- Análise dos Dados: Após a exportação do arquivo .cxl o algoritmo irá realizar o tratamento dos dados, essa fase será subdivida em: Análise dos Conceitos e Estrutura da Proposição onde será levado em consideração a análise sintática e semântica dos conceitos, para essa etapa utilizaremos a biblioteca Apache OpenNLP, uma biblioteca Java apoiada em aprendizado de máquina para o processamento de linguagem natural, dentre as tarefas abordadas pela OpenNLP podemos citar: tokenização, reconhecimento de entidades nomeadas, extração de sintagmas e análise sintática. Para realizar a análise contextual das proposições extraídas se faz necessário a utilização de indexadores automáticos que utilizam critérios sintático-semânticos denominados parser, utilizando-se nessa análise de uma base de dados gramatical preestabelecida e com seu conteúdo em língua portuguesa. Para essa tarefa podemos citar a ferramenta Tropes que é capaz de detectar palavras que tem a mesma representatividade na frase, resolvendo problemas de ambiguidade com taxa média de erro de 5\%, verificando assim o contexto da proposição realizada pelo aluno [OTHERO and MENUZZI 2005], é nesta fase que os conceitos obrigatórios são revisados. Profundidade da Estrutura Hierárquica, por se tratar de um grafo, os mapas conceituais podem ser analisados em nível de profundidade dos seus vértices, conexão e reconexão das suas arestas, podendo ter um peso numérico associado, sendo neste momento que a 
VII Congresso Brasileiro de Informática na Educação (CBIE 2018)

Anais do XXIX Simpósio Brasileiro de Informática na Educação (SBIE 2018)

análise de vizinhança é realizada.

- Ranqueamento: Após a conclusão das fases anteriores, é processado o resultado de todas as análises, baseando-se nas rubricas dos critérios de análise de mapas conceituais. Esse resultado é visualizado em um ambiente web onde o professor terá acesso aos resultados compilados.

\section{Conclusão}

Esse trabalho é parte inicial do projeto de conclusão do Mestrado em Ciência da Computação e, por se tratar de uma proposta, ainda não obtivemos resultados do experimento, bem como há a necessidade de aprofundamento maior nos conceitos e técnicas aqui expostos. Os trabalhos futuros estão direcionados ao desenvolvimento da aplicação, as abordagens em torno das técnicas de processamento de linguagem natural e melhorias na estruturação do ranqueamento do mapa conceitual.

\section{Referências}

Ausubel, D. P., Novak, J. D., and Hanesian, H. (1980). Psicologia educacional. Interamericana.

Cañas, A., Novak, J., and Vanhear, J. (2012). Concept map-based knowledge assessment tasks and their scoring criteria: An overview.

Cicuto, C. A. T., Correia, P. R. M., et al. (2012). Análise de vizinhança: uma nova abordagem para avaliar a rede proposicional de mapas conceituais. Revista Brasileira de Ensino de Física, 34(1).

Krathwohl, D. R. (2002). A revision of bloom's taxonomy: An overview. Theory into practice, 41(4):212-218.

Moreira, M. A. (2012). Mapas conceituais e aprendizagem significativa (concept maps and meaningful learning). Aprendizagem significativa, organizadores prévios, mapas conceituais, digramas $V$ e Unidades de ensino potencialmente significativas, page 41.

OTHERO, G. d. Á. and MENUZZI, S. d. M. (2005). Linguística computacional: teoria e prática. São Paulo: Parábola.

Trevelin, A. T. C., Pereira, M. A. A., and de Oliveira Neto, J. D. (2013). A utilização da "sala de aula invertida" em cursos superiores de tecnologia: comparação entre o modelo tradicional e o modelo invertido "flipped classroom" adaptado aos estilos de aprendizagem. Journal of Learning Styles, 6(12).

Valente, J. A. (2014). Blended learning e as mudanças no ensino superior: a proposta da sala de aula invertida. Educar em Revista, (4).

Wang, M. M., Yang, S. J., Okamoto, T., Nagata, N., and Anma, F. (2011). Knowledge management \& e-learning: An international journal (km\&el). 